\section{P1-28 LINKAGE OF PRIMARY AND SECONDARY CARE DATA TO IDENTIFY RISK FACTORS FOR EMERGENCY HOSPITAL ADMISSION FOR COPD: NEGOTIATING THE LEGAL AND ETHICAL HURDLES}

doi:10.1136/jech.2011.142976c.22

${ }^{1} \mathrm{~L}$ Hunter, ${ }^{*}{ }^{2} \mathrm{C}$ Fischbacher, ${ }^{1,3} \mathrm{~S}$ Wild, ${ }^{1} \mathrm{R}$ Hardie. ${ }^{1} \mathrm{NHS}$ Lothian, Edinburgh, UK; ${ }^{2}$ Information Services Division, NHS Scotland, Edinburgh, UK; ${ }^{3}$ University of Edinburgh, Edinburgh, UK

Introduction We aimed to identify modifiable risk factors in primary care associated with emergency hospital admissions with Chronic Obstructive Pulmonary Disease in Lothian, Scotland. We sought permission to obtain data from primary to secondary care databases in a way that would allow the required analyses while ensuring that patient confidentiality was protected.

Methods The research protocol proposed an "anonymised linkage" method that linked personal identifiers and pseudonymised them before adding clinical data. All identifiers would be removed from the final analysis dataset. Consent to participate in the project was sought from general practitioners. General information was available to patients about secondary uses of their personal data. Ethical approval was sought from relevant local to national committees.

Results Conflicting views were expressed by different ethical committees. Some took the view that individual patient consent was required for the proposed approach while others viewed it as anonymous database research.

Conclusions In large epidemiological studies it may not be practicable to obtain individual patient consent. UK law and professional guidance supports the use of patient data for research without consent in some circumstances provided the research is deemed to be in the public interest. Data linkage is a powerful tool increasingly used for epidemiological research. However, its use to improve health in Scotland is hampered by lack of clear guidance and inconsistent interpretation. While it is important that patient confidentiality is respected, a balance needs to be struck to enable use of patient data to improve health and the quality of care.

\section{P1-29 USING INTERNATIONAL EVIDENCE TO DEVELOP A CONCEPTUAL FRAMEWORK OF FACILITATORS AND BARRIERS OF CHLAMYDIA SCREENING SERVICE IN COMMUNITY PHARMACIES}

doi:10.1136/jech.2011.142976c.23

M Z Kapadia, * P Warner, K Fairhurst. Centre for population Health sciences, University of Edinburgh, Edinburgh, UK

Introduction Community pharmacies have recently become more involved in providing chlamydia testing and treatment CT\&T. We have reviewed published evidence and developed a conceptual framework regarding facilitators of and barriers to access and provision of chlamydia testing and treatment in community pharmacies. Methods Electronic databases were searched using the keywords and grey literature was solicited from experts. Studies selected were imported to NVIVO8 and thematic analysis was performed to identify key facilitators and barriers to acceptability/success of CT\&T community pharmacy service. The emerging themes were categorised to develop a conceptual framework.

Results 17 papers and reports were included. The framework developed suggests barriers and facilitators operate at three conceptual levels; i) Service delivery, ii) Young people's decision to access service; and iii) Policy stakeholders' strategies. Some factors operate at more than one level. Key service user factors identified were convenience of access (location, no need for appointment and extended opening hours for pharmacies), pharmacy visits free of social stigma, within-pharmacy facilities for example, counselling area and toilet, level of privacy and confidentiality and self-perceived risk of chlamydia. Identified barriers at provider level were, insufficient promotion of service, staff workload and untrained staff. Policy stakeholders' strategies of implementing services in deprived areas, health professionals' relationship with pharmacy staff and incentives were considered key to the success.

Conclusion Chlamydia screening in community pharmacies is broadly acceptable to both service users and providers. However if screening is to succeed, policy makers must accommodate the facilitators and barriers identified by young people and pharmacy staff.

\section{P1-30 CONFOUNDED CLUSTERS: AN ASSESSMENT OF THE ADEOUACY OF MEASURES TO MINIMISE CONFOUNDING IN CLUSTER RCTS}

doi:10.1136/jech.2011.142976c.24

${ }^{1} S$ V Katikireddi, ${ }^{2}$ J Thoulass, ${ }^{3} \mathrm{C}$ Bonell, ${ }^{1} \mathrm{~L}$ Bond. ${ }^{1} \mathrm{MRC}$ Social and Public Health Sciences Unit, Glasgow, UK; ${ }^{2}$ Section of Population Health, University of Aberdeen, Aberdeen, UK; ${ }^{3}$ Department of Social and Environmental Health Research, London School of Hygiene and Tropical Medicine, London, UK

Introduction Cluster randomised controlled trials (CRCTs), where the unit of allocation is a group, are particularly useful in Public Health, when interventions are targeted at populations. Randomisation is the 'gold standard' for dealing with both known and unknown confounders as these are balanced between comparison groups with sufficient numbers randomised. However, CRCTs may have few units randomised, increasing the likelihood of confounding. Confounding can be minimised by design (eg, minimisation) or analysis. We investigated methods used to reduce confounding and their adequacy in a sample of alcohol prevention CRCTs.

Methods Existing systematic reviews published in the Cochrane database assessing efficacy/effectiveness of primary/secondary prevention methods in young people to reduce alcohol use/harms were identified. Reporting of the following for all included CRCTs was established: baseline cluster characteristics, type of randomisation, participation rate, attrition rate and measures reducing confounding at analysis. All data were collected using standardised forms by two independent reviewers with discrepancies resolved with a third reviewer

Results Two Cochrane systematic reviews were identified yielding a total of 30 CRCTs. Baseline measures at the cluster level were not always reported and the potential for confounding was often not considered in design, analysis and interpretation of results.

Conclusion To our knowledge, this is the first study that investigates whether researchers adequately consider confounding at a clusterlevel in CRCTs. We recommend greater adherence to the CONSORT extension for CRCTs with explicit consideration of confounders during all stages of research. This helps ensure misleading effectiveness estimates are not used for Public Health decisions.

\section{P1-31 CLINICAL USEFULNESS OF FRAMINGHAM CARDIOVASCULAR RISK PROFILE DURING A 10-YEAR FOLLOW-UP IN IRAN}

doi:10.1136/jech.2011.142976c.25

${ }^{1,2} \mathrm{D}$ Khalili, ${ }^{*}{ }^{1} \mathrm{H}$ Soori, ${ }^{2} \mathrm{~F}$ Hadaegh, ${ }^{2} \mathrm{~F}$ Azizi. ${ }^{1}$ Department of Epidemiology, School of public health, Shahid Beheshti University of Medical Sciences, Tehran, Iran; ${ }^{2}$ Prevention of Metabolic Disorders Research Center, Research Institute for Endocrine Sciences, Shahid Beheshti University of Medical Sciences, Tehran, Iran

Introduction Clinical usefulness of a risk function is a new concept that should be considered beyond the traditional checking of 
discrimination and calibration for performance of a model. Framingham risk score (FRS) for cardiovascular disease is a widely used one which has been validated in different countries but its clinical usefulness has been neglected.

Methods We checked discrimination of FRS and so its calibration and clinical usefulness before and after recalibration in a population based cohort, Tehran lipid and glucose study, of 2640 men and 3584 women aged $30-74$ years. To check clinical usefulness, we used decision curve analysis (DCA) and calculated net benefit of treatment for patients with $\geq 20 \%$ of 10 year probability of disease according to FRS model.

Results The area under the curve for FRS model, was 0.794 and 0.838 for men and women respectively. The original model had a poor calibration but got a good one after recalibration (HosmerLemeshow $\chi^{2}$ statistic of 16.8 for men and 18.4 for women). Based on DCA, FRS was clinically useful in cut points of $10 \%-30 \%$, as threshold probability of disease that a patient should be treated, before and after recalibration. The net benefit of model to treat patients at cut point of $20 \%$ did not differ significantly before and after recalibration in both men and women ( $p>0.3$ based on bootstrap resampling).

Conclusion Original FRS has a good discrimination and poor calibration in Iran but considering clinical usefulness, it can be used even without recalibration.

\section{P1-32 TESTING LIFE COURSE MODELS TO INVESTIGATE THE EFFECT OF SOCIOECONOMIC POSITION ON CRYSTALLISED COGNITIVE FUNCTION IN OLDER AGE, ACCOUNTING FOR MISSING DATA}

doi:10.1136/jech.2011.142976c.26

\begin{abstract}
${ }^{1,2} \mathrm{R}$ Landy, ${ }^{1} \mathrm{~J} \mathrm{Head},{ }^{2} \mathrm{M}$ Richards, ${ }^{2} \mathrm{R}$ Hardy. ${ }^{*}$ Department of Epidemiology and Public Health, University College London, London, UK; ${ }^{2}$ MRC Unit for Lifelong Health and Ageing, Department of Epidemiology and Public Health, London, UK
\end{abstract}

Objective To investigate how lifetime socioeconomic position (SEP) is associated with later-life crystallised cognitive function, accounting for different missing data mechanisms.

Participants A nationally representative population sample born in 1946 (MRC National Survey of Health and Development; NSHD, $\mathrm{N}=5362$ ), and a sample of British civil servants (Whitehall II; WHII, $\mathrm{N}=10308$ ).

Methods Novel structured statistical approach to distinguish between accumulation and sensitive period life course models using SEP measures from childhood, early-adulthood and midlife. Results of complete case (CC) (assuming missing completely at random), multiple imputation (MI) (missing at random) and a Heckman selection model (missing not at random) were compared.

Outcomes National Adult Reading Test, age 53 (NSHD); Mill Hill Test, age 55-79 (WHII)

Results NSHD: After adjusting for childhood cognitive function, the best fitting model was an accumulation model allowing SEP at each time point to have its own estimate. However estimates varied by missing data method (women: childhood SEP: CC: coefficient $=1.11$ (95\% CI 0.15 to 2.06 ), MI: coefficient $=1.82$ (95\% CI 0.87 to 2.76 ), Heckman: coefficient $=0.70$ (95\% CI -0.38 to 1.78$)$ ). WHII (not adjusted for childhood cognition): the best fitting model represented accumulation in adulthood only, with childhood SEP not significant. Conclusion Despite adjustment for childhood cognitive score, childhood SEP remains important in NSHD, whereas in Whitehall II childhood SEP was not associated with cognitive function. These differences may be due to recall bias of early SEP in WHII. Our findings demonstrate the utility of the method for distinguishing models of how SEP across the life course influences cognition and the importance of dealing with missing data.

\section{P1-33 ADULT DNA METHYLATION IN RELATION TO PRENATAL NUTRITION AND RISK FACTORS FOR CARDIO-VASCULAR DISEASE}

doi:10.1136/jech.2011.142976c.27

L H Lumey, ${ }^{*}$ M B Terry, L Delgado-Cruzata, K Gonzales, 0 Wang, Y Liao, E Susser, I McKeague, R Santella. Columbia University, New York, USA

Epigenetic changes may play an important role in the development of adult disease. Exposure to a prenatal famine environment has been associated with a persistent decrease in DNA methylation of the IGF2 gene, and other early life factors like birthweight with adult genomic DNA methylation. We evaluated genomic DNA methylation in relation to prenatal nutrition and CVD risk factors. Our study includes 353 births from three clinics with prenatal exposure to the Dutch famine of 1944-1945, 296 before-or-after the famine births as unexposed time controls, and 311 same-sex siblings of either group as unexposed family controls. All study subjects underwent medical examinations and DNA collections at a mean age of $\sim 58$ years. We used Luminometric methylation (LUMA) and LINE-1 (Long Interspersed Nucleotide Element 1) pyrosequencing assays to quantify genomic DNA methylation. Mean DNA methylation by LUMA was 75.5\% (SD 2.2) and by LINE-1 77.1\% (SD 2.5). Neither was associated with prenatal famine exposure. Using all controls, famine exposure showed a decline of $0.12 \%(95 \% \mathrm{CI}$ -0.42 to $0.18 ; p=0.44)$ by LUMA and an increase of $0.05 \%(-0.33$ to $0.21 ; \mathrm{p}=0.68)$ by LINE- 1 . Using family controls, the increases were $0.36 \%$ (LUMA) and $0.16 \%$ (LINE-1). Neither assay was associated with adult cholesterol, blood pressure, body size, type 2 diabetes mellitus, gender or age. Our results suggest that these genomic DNA methylation markers may not be associated with prenatal famine; further work should target regions in the genome that may be differentially methylated in response to early life exposures.

\section{P1-34 CAN ASSOCIATIONS BETWEEN PRENATAL NUTRITION AND BODY MASS INDEX BE EXPLAINED BY COMIMON GENETIC VARIANTS? FINDINGS FROM THE DUTCH HUNGER WINTER FAMILIES STUDY}

doi:10.1136/jech.2011.142976c.28

${ }^{1}$ L H Lumey, ${ }^{* 2} \mathrm{~A}$ Vaarhorst, ${ }^{3} \mathrm{~A} D$ Stein, ${ }^{2} \mathrm{~B}$ Heijmans. ${ }^{1}$ Columbia University, New York, USA; ${ }^{2}$ Leiden Univ Med Ctr, Leiden, The Netherlands; ${ }^{3}$ Emory University, Atlanta, Georgia, USA

Genome wide association (GWAS) studies have identified single nucleotide polymorphisms (SNPs) related to body mass index (BMI $\mathrm{kg} / \mathrm{m}^{2}$ ). Associations have also been reported between fetal malnutrition and BMI. We use the circumstances of the Dutch Hunger Winter of 1944-1945 to further examine these relations. We studied 348 adult men and women born in affected cities in the western Netherlands who had been exposed to famine during pregnancy, 294 born before or after the famine as time-controls, and 305 same-sex unexposed siblings of above groups as family controls. Mean age at examination was 58 years. We evaluated common variants in the FTO, TMEM18, MC4R, GNPDA2, BDNF, SEC16B, NEGR1, SH2B1, SFRS10, MTCH2, and KCTD15 genes related to BMI. A genetic risk score was calculated for each individual by summing the number of risk alleles in these genes. Scores were also weighted using recent GWAS estimates of gene specific changes in BMI per risk allele. Institutional ethics committees gave the appropriate approvals for the study. Genetic risk scores had a mean of 11.4 (SD 2.2) and were not related to prenatal famine. Adult BMI was 1.34 units higher among famine exposed (95\% CI 0.73 to 1.94 ) and further adjustments for risk score had little effect. There was no statistical 